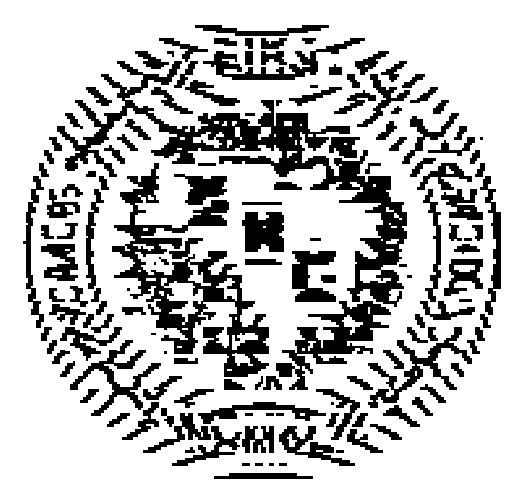

Normatização para a Concessão e Revalidação do Título de Especialista em Cardiologia e de Área de Atuação dentro da Especialidade 


\title{
Normatização para a concessão e revalidação do título de especialista em cardiologia e de área de atuação dentro da especialidade
}

\author{
Editor \\ Miguel Antonio Moretti (SP) \\ Membros \\ Benedito Carlos Maciel (SP), \\ Marcos Vinicius Bolivar Malachias (MG), \\ Roberto Pozzan (RJ), \\ Dalton Bertolim Precoma (PR), \\ Antônio Silveira Sbissa (SC), \\ Sergio Zimmermann (SC), \\ Walter Geraldo da Silveira (DF), \\ Gil Eduardo Perini (GO), \\ Frederico Augusto Lima e Silva (CE), \\ João David de Sousa Neto (CE), \\ Brivaldo Markmann Filho(PE), \\ Sadi de Carvalho (AL), \\ Aristoteles Conte de Alencar Filho (AM)
}

Coordenador do projeto diretrizes

Jorge Ilha Guimarães (RS)

Revisão e colaboração:

todos os presidentes das regionais e de departamentos

Aprovada pela Associação Médica Brasileira (AMB) 


\section{Normatização}

A especialidade em Cardiologia Clínica é reconhecida pela Comissão Mista de Especialidades: Associação Médica Brasileira (AMB), Conselho Federal de Medicina (CFM) e Comitê Nacional de Residência Médica (CNRM) [resolução CFM no 1634/2002 - J Conselho Federal Medicina Ano XVII No 134 maio/junho/2002 e JAMB ano $43 n^{\circ} 1317$ Jan/Fev 2002].

O título de Especialista em Cardiologia é fornecido pela AMB (através da Sociedade Brasileira de Cardiologia-SBC) e registrado pelo CFM [resolução CFM $n^{\circ} 1286 / 89$ ], segundo convênio firmado entre a AMB e a SBC em 01/07/2002. $\mathrm{Na}$ SBC, a assessoria em assuntos referentes ao título de especialista e aos certificados de áreas de atuação é dada pela Comissão Julgadora do Título de Especialista em Cardiologia (CJTEC), nos termos do Anexo C desta Normatização.

\section{Objetivo}

O Título de Especialista em Cardiologia (TEC) tem como objetivo distinguir o profissional médico apto a exercer a especialidade de Cardiologia Clínica, que tenha apresentado formação médico-científica adequada, de acordo com a SBC e a legislação vigente.

\section{Pré-requisitos} deverá:

O Candidato ao Título de Especialista em Cardiologia

1. estar formado há, pelo menos, 03 (três) anos, em Faculdade de Medicina reconhecida pelo Ministério da Educação e Cultura (MEC) e encontrar-se regularmente inscrito no Conselho Regional de Medicina (CRM) de seu local de trabalho;

2. apresentar comprovação de:

a - ter concluído o último ano de residência em Cardiologia Clínica, credenciada pela Comissão Nacional de Residência Médica, desenvolvida em período integral, com mínimo de 2 anos completos de duração;

\section{OU}

b - ter concluído o último ano de estágio em Cardiologia Clínica, com programa semelhante ao exigido naresidência [resolução CNRMn ${ }^{\circ} 1$ de 14/05/2002], desenvolvidoemperío do integral, com mínimo de 2 anos completos de duração;

\section{OU}

c. mínimo de 5 anos de atividade hospitalar como cardiologista clínico*;

3. apresentar carta de recomendação, com firma reconhecida, de três sócios da SBC (portadores do TEC), atestando atividade contínua, até os dias atuais, do candidato na área de cardiologia clínica (modelo anexo A).

Os comprovantes (cópias autenticadas) deverão ser encaminhados juntamente com a ficha de inscrição.

*Apresentar os documentos comprobatórios, sob pena de ter anulada a prova do título.

\section{Da realização das provas}

Os candidatos ao TEC deverão se submeter à prova de conhecimento teórico e, também, à prova prático-oral, quando não preencherem os critérios de isenção desta última.

Para tanto, deverão:

1.estar inscritos para a prova anual do TEC, dentro dos prazos estabelecidos, tendo pago a taxa de inscrição;

2.cumprir todas as normas estabelecidas pela SBC;

3. comparecer e realizar a prova no local e hora determinados.

\section{Daprova teórica}

1.A prova anual constará de 120 (cento e vinte) questões de múltipla escolha e será elaborada por empresa especializada e independente, sob a supervisão dos membros da CJTEC. As questões versarão sobre os temas gerais da cardiologia, com vistas à verificação da boa formação de um especialista em cardiologia clínica.

2.Serão enfocados, prioritariamente, aspectos práticos da especialidade, com base em livro texto consagrado de cardiologia (E. Braunwalde W. Hurst)e, principalmente, nas últimas Diretrizes publicadas da SBC.

3. A prova será composta por 100 (cem) questões com valor de 01 (um) ponto e 20 (vinte) questões com valor de 03 (três) pontos, sendo 160 (cento e sessenta) o valor máximo dos pontos possíveis.

\section{Prova prática/oral}

1. Só estarão habilitados para se submeter à prova prática os candidatos que forem aprovados na prova teórica.

2. Os candidatos serão submetidos à verificação do conhecimento e das habilidades práticas relativas à cardiologia clínica.

3. A prova será aplicada por banca examinadora constituída por indicação da CJTEC da SBC, composta por 03 (três) membros, todos portadores de título de especialista em cardiologia, sendo realizada em local definido pela CJTEC.

4. Na composição da banca, prioritariamente, 02 (dois) de seus membros deverão ser de locais diferentes daquele em que for realizada a prova e um dos membros deverá, preferencialmente, pertencer à CJTEC.

5. O candidato poderá se submeter à nova prova prática sem pagamento de taxa de inscrição, caso não seja aprovado na primeira vez. A eventual não aprovação na segunda prova prática implicará a necessidade de realização de um novo conjunto de provas (teórica e prática), quando haverá obrigatoriedade de pagamento de nova taxa de inscrição.

\section{Dos critérios de avaliação}

Serão considerados aprovados os candidatos que:

1.obtiverem $60 \%$ do total de pontos da prova ou um total de pontos que os posicione acima do percentil de $70 \%$, seguindo-se, em cada prova, o critério de selecionar o maior número de candidatos inscritos;

\section{$\mathbf{E}$}

2. obtiverem uma nota média igual ou superior a 7,0 (sete) na prova prática/oral. 


\section{Observações}

I. Estarão isentos da realização da prova prática/oral os candidatos que comprovarem:

a- um ano de residência em Clínica Médica, desenvolvida em período integral, aprovada pelo CNRM;

\section{OU}

b - um ano de estágio desenvolvido em período integral em Clínica Médica, nos mesmos padrões estabelecidos pelo CNRM [ resolução CNRMn ${ }^{\circ} 1$ de 14/05/2002];

\section{OU}

c- dez anos de atividade hospitalar como cardiologista clínico*.

II. Após a definição do critério a ser utilizado para estabelecer a nota mínima para aprovação na prova teórica, poderão ser adicionados pontos aos obtidos na prova escrita pelo candidato, de acordo com a tabela abaixo:

Os comprovantes (cópias autenticadas) deverão ser encaminhados juntamente com a ficha de inscrição.

III. Os estrangeiros candidatos ao TEC da SBC/AMB deverão: 1) estar devidamente inscritos no CRM de seu estado de atuação há, no mínimo, 3 anos; 2) comprovar Residência ou Estágio equivalente em Cardiologia Clínica de, no mínimo, 2 anos e 3 ) obrigatoriamente, realizar as provas escrita e prática.

\section{Normas para Revalidação do Título de Espe- cialista em Cardiologia - TEC}

I. O título de Especialista em Cardiologia deverá ser revalidado a cada período de cinco anos, ou seja, ao final de cinco anos, seu portador deverá revalidá-lo por mais cinco anos.

- Para os já portadores do TEC desde antes de dezembro de 2000, o quinquênio para revalidação para os próximos cinco anos teve início em 01 de janeiro de 2001;

- Para os que adquiriram otítulo após dezembro de 2000, operíodo de cinco anos passará a ser contatado a partir da data de emis são da declaração de aprovação fornecida pela SBC.

\begin{tabular}{|lcc|}
\hline \multicolumn{2}{|c|}{ Característica } & Pontos \\
\hline Livre-Docência em cardiologia clínica & $50^{*}$ \\
Doutorado (tese defendida com tema em cardiologia clínica) & $30^{*}$ \\
Mestrado (dissertação defendida com tema em cardiologia clínica) & $15^{*}$ \\
Tempo de Formado (análise do currículo) & $10-15$ anos & 04 \\
& $15-20$ anos & 06 \\
& $>20$ anos & 08 \\
\hline * Somam-se apenas os pontos da maior titulação, não acrescentado, neste caso, \\
\hline
\end{tabular}

II. A revalidação poderá ser conseguida através de uma das duas modalidades:

Modalidade A - Comprovação da aquisição de,pelo menos, 100 pontos em atividades de educação continuada na área de cardiologia clínica ao longo do quinquênio, discriminados conforme as tabelas abaixo. Os pontos não poderão ser transferidos de um período para outro.

Modalidade B - Através de prova a ser realizada no último ano do quinquênio. A nota mínima para aprovação será igual a 50\% do total de pontos. (Obs.: Será a mesma prova para a obtenção do TEC)

III. Ao solicitar a revalidação, o sócio da SBC deverá estar quite com suas obrigações societárias. No caso de não ser sócio, o candidato deverá pagar taxa específica junto à SBC.

Quadros de Pontuação para Revalidação (ver anexo B)

\section{Área de Atuação Dentro da Especialidade}

Até o presente momento, as Áreas de Atuação dentro da Cardiologia, reconhecidas pela Comissão Mista de Especialidades, são: Cardiologia Pediátrica (também é área de atuação da pediatria), Ecocardiografia, Eletrofisiologia Clínica Invasiva, Ergometria e Hemodinâmica e Cardiologia Intervencionista [Resolução 1966 de 2003, Jornal do Cremesp - 192 Agosto 2003].

O certificado é fornecido pela AMB (através da Sociedade Brasileira de Cardiologia - SBC, representada pela CJTEC) e registrado pelo CFM.

Farão jus ao certificado das Áreas de Atuação descritas, os médicos que forem aprovados em concurso promovido pelo respectivo Departamento e possuírem o título de especialista em Cardiologia.

A regulamentação do Concurso para obtenção do Certificado de Área de Atuação deverá seguir os princípios básicos desta Normatização e ser aprovada pela CJTEC, que será o órgão encarregado da interface entre os departamentos e a SBC/AMB.

\section{Disposições Gerais}

I. Todos os procedimentos necessários para o cumprimento da presente Normatização ficarão a cargo da Central de Eventos da SBC.

II.Estas normas entrarão em vigor a partir da data de sua divulgação no site da SBC, quando então serão encaminhadas mensagens para todos os sócios; a divulgação in-

\begin{tabular}{|lc|}
\hline Participação docente em atividades científicas & Aulas em cursos, mesas redondas, palestras, conferências, ou similares*** \\
\hline Congressos da SBC e Curso Nacional de Reciclagem & 05 \\
Congresso Regional e de Departamento & 03 \\
Congresso Internacional de Sociedades Federadas & 10 \\
Outras atividades da programação científica oficial da SBC & 02 \\
\hline ***Aulas ministradas em qualquer atividade oficial da SBC, por convite da comissão científica, com exceção das funções de presidente ou secretário de conferência, \\
moderador ou coordenador de mesa redonda ou simpósio e instrutor em cursos ACLS/BLS/PALS/PBLS. \\
\hline
\end{tabular}


cluirá outros links na rede mundial de computadores e o jornal da SBC. Posteriormente, serão publicadas nos Arquivos Brasileiros de Cardiologia - ABC.

III. Estas diretrizes só poderão ser modificadas após período mínimo de 3 anos; as emendas que se fizerem necessárias, devido a mudanças nas regras de instâncias superiores à CJTEC (SBC, AMB, CFM, por exemplo), não poderão ser feitas com intervalos menores que 6 meses, devendo ser solicitadas pela CJTEC e aprovadas pela diretoria da SBC.

\begin{tabular}{|llcc|}
\hline & Publicações em Cardiologia & Autor & Co-autor \\
\hline \multirow{2}{*}{ Artigos } & Arquivos Brasileiros de Cardiologia & 15 & 08 \\
originais & Outras revistas indexadas & 06 & 03 \\
& Outras línguas/ inglês (Index Medicus) & 15 & 08 \\
Artigos de & Arquivos Brasileiros de Cardiologia & 08 & 04 \\
Revisão & Outras revistas indexadas & 03 & 02 \\
& Outras línguas/ inglês (Index Medicus) & 08 & 04 \\
& Arquivos Brasileiros de Cardiologia & 03 & - \\
Editorial & Outras revistas indexadas & 01 & - \\
& Outras línguas/ inglês (Index Medicus) & 03 & - \\
Capítulo de livro científico em Cardiologia & 05 & 03 \\
Editoria de livros científicos em Cardiologia & 30 & - \\
\hline
\end{tabular}

IV. Esta Normatização, mesmo após modificação, deverá se submeter à aprovação, por escrito, da AMB.

V.As situações aqui omissas serão julgadas e decididas pela CJTEC, com base na aprovação de, pelo menos, dois terços de seus membros.

VI. Esta normatização entrará em vigor a partir da data de sua publicação pelos órgãos oficiais da Sociedade Brasileira de Cardiologia, anulando todas as outras disposições anteriores.

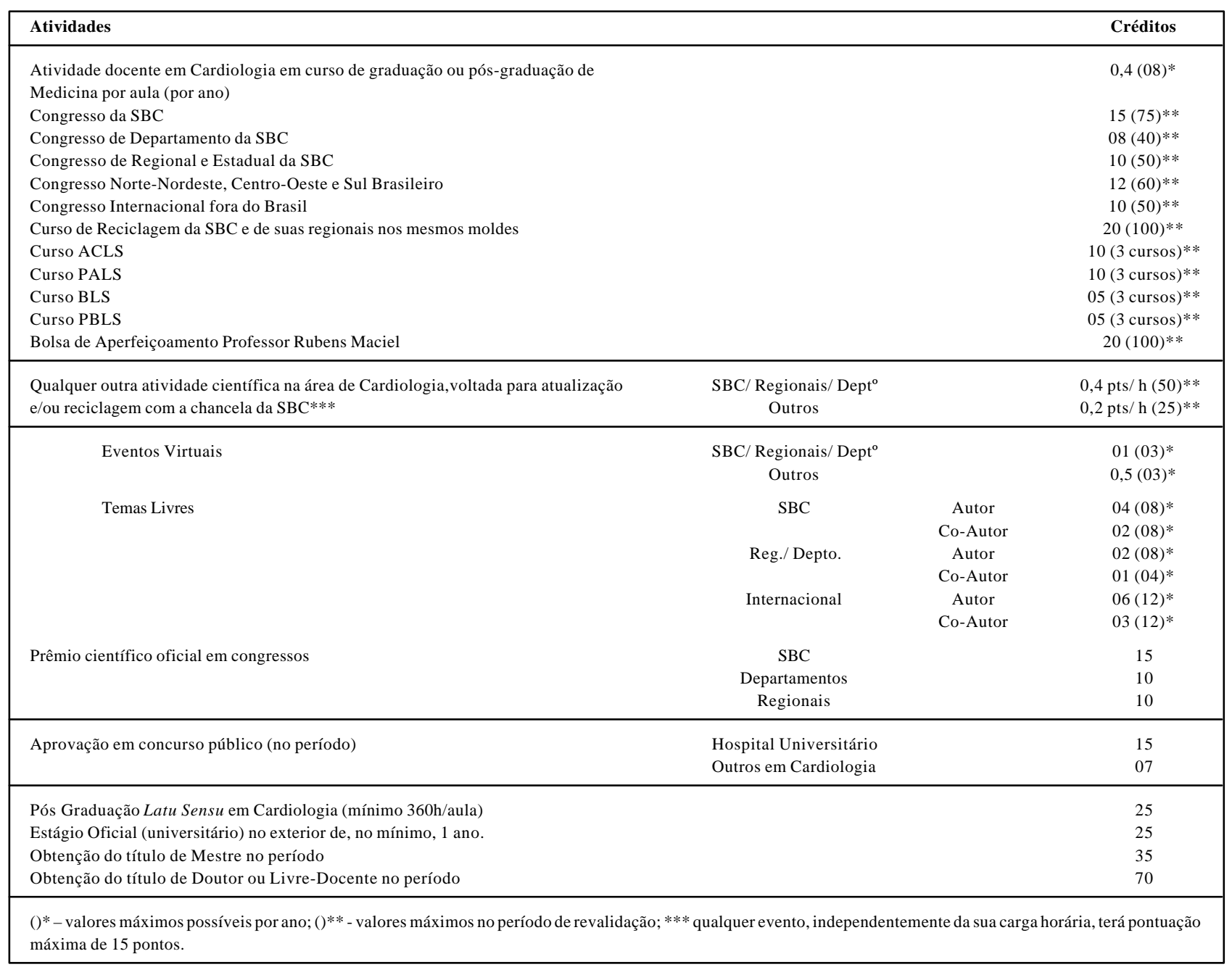


Anexo B - Normas para Inclusão de Eventos na Agenda Científica Oficial da SBC

Para inclusão na Agenda Científica Oficial da SBC, bem como para pontuação, tal como previsto nas NoRMATIZAÇÃo PARA CONCESSÃo E REVALIDAÇÃo DO TíTULO DE ESPECIALISTA EM CARDIOLOGIA, os cursos e outras atividades científicas voltadas para atualização e/ou reciclagem em cardiologia clínica deverão estar de acordo com as seguintes regras:

1. Os eventos deverão ser encaminhados à CJTEC, para análise, até 30 de setembro (quando a realização estiver prevista para o ano subseqüente) e 31 de março (quando sua realização estiver prevista para o segundo semestre do mesmo ano). ESSAS DATAS SÃO LIMITES E NÃO PRORROGÁVEIS.

2. O encaminhamento deverá ser feito mediante preenchimento completo do formulário específico on-line, disponível no site da SBC (http://educacao.cardiol.br/ titulo/cadastro_eventos.asp). Quando isso não acontecer poderá haver retardo da análise e aprovação do evento, podendo resultar em não pontuação do mesmo e, ainda, em perda da ordem de prioridade.

3. O encaminhamento à CJTEC deverá se dar, preferencialmente, através das Regionais ou Departamentos; nesse caso, o conteúdo científico do evento deverá ter o aval do presidente ou diretor científico da Regional ou Departamento, cuja identificação deverá ser incluída juntamente com o programa.

4. O Congresso Brasileiro de Cardiologia, promovido pela SBC, bem como alguns cursos específicos, com pontuação prevista na NORMATIZAÇÃo PARA CONCESSÃo E REVALIDAÇÃO DO TíTULO DE ESPECIALISTA EM CARDIOLOGIA, farão parte da agenda científica oficial da SBC, sem necessidade de sua programação ser submetida à análise da CJTEC.

5. Não serão incluídos nesta agenda, e, conseqüentemente, não receberão pontuação com vistas à revalidação de título de especialista: 1) reuniões científicas ou encontros periódicos de clínicas ou hospitais; 2) eventos que ocorrerem dentro dos congressos, ou que constem de sua programação oficial.

6. Os eventos que não forem submetidos à CJTEC ou que não tenham sidos aprovados por esta comissão ou, ainda, que tenham sido cadastrados fora do prazo, mesmo que sejam de Regionais ou Departamentos da SBC, não constarão na referida agenda e não somarão pontos para revalidação do título de especialista.

7. Para evitar a superposição de datas, é necessário que se informe, além da data prevista, uma segunda opção, que, na hipótese de vir a ser utilizada, passará a ser (com a concordância do organizador do evento) a data oficial do evento.

8. No programa do evento, deverá constar, de forma clara, a carga horária real (tempo de aula corrido, ou seja, tempo total da atividade menos o tempo dos intervalos), a data, o local e os temas.

9. Em caso de coincidência de eventos na mesma data e local, terá prioridade, para inclusão na agenda, o evento encaminhado por Regional ou Departamento, ou aquele que foi encaminhado com maior antecedência.

10. Em relação a eventuais mudanças nas datas ou nos programas dos eventos, após aprovação pela CJTEC,esta modificação somente poderá ser efetivada mediante consulta prévia à CJTEC, com antecedência mínima de 45 dias antes da data oficial do referido evento. A não observância desta norma implicará retirada do evento do calendário oficial e não pontuação do mesmo com vistas à revalidação do TEC.

11. Para que o evento (todos, exceto congressos da SBC, de departamentos e de regionais) possa ser efetivamente pontuado, o coordenador/responsável deverá encaminhar à CJTEC, por via eletrônica, o programa definitivo até quinze dias antes da realização do evento e, até trinta dias depois, preencher formulário específico no site da SBC, com dados relativos ao evento. Essas informações serão utilizadas em processos de auditoria conduzidos pela CJTEC.

12. Todos os participantes do evento deverão receber, ao seu final, um certificado, no qual deverá constar o ID fornecido pela CJTEC, com a assinatura do responsável e, no caso de Regionais ou Departamentos, com a assinatura do diretor científico ou presidente.

13. Os congressos oficiais de Departamentos, Regionais e Macrorregiões (apenas um por entidade) contam, respectivamente, 8,10 e 12 pontos, independentemente da programação, ou seja, estas atividades têm pontuação fixa. Para sua aprovação, entretanto, será necessário o encaminhamento do pré-programa, juntamente com o formulário preenchido on-line

14. Serão considerados "eventos virtuais" os cursos de atualização ou reciclagem em Cardiologia, realizados via Internet, contendo questionários de avaliação de aproveitamento.

a.A inscrição para validação processar-se-á de forma habitual, não sendo, contudo, necessário discriminar a carga horária.

b.Para cada evento, será necessária a elaboração de um questionário, com, no mínimo, 50 questões, a ser apresentado pelo organizador. O organizador deverá informar aos alunos o ID do evento para que os mesmos respondam ao questionário, diretamente no Portal da SBC, na rede mundial de computadores. Serão considerados aprovados e farão jus aos pontos da revalidação os alunos que acertarem $70 \%$ das questões, numa única tentativa.

15. Os coordenadores e/ou instituições responsáveis pelos eventos que, por ventura, não cumprirem as determinações acima e os programas previstos estarão sujeitos a sanções estabelecidas pela CJTEC.

\section{Disposições Gerais}

I. As normas sobre inclusão de eventos que contarão pontos para o processo de revalidação, na agenda oficial da SBC, serão publicadas em separado no Jornal da SBC e também ficarão à disposição no site da SBC na rede mundial de computadores, podendo ser modificadas somente por solicitação da CJTEC e com aprovação, por maioria simples, dos presidentes de regionais e departamentos, através de consulta por via eletrônica.

II. Todos os procedimentos necessários para o cumprimento destas normas ficarão a cargo da Central de Eventos da SBC.

III.Estas normas entrarão em vigor a partir da data de sua divulgação no site da SBC, quando, então, serão encaminhadas mensagens para todos os sócios. A divulgação incluirá outros links na rede mundial de computadores e o jornal da SBC. Posteriormente, serão publicadas nos Arquivos Brasileiros de Cardiologia - ABC.

IV. As situações aqui omissas serão julgadas e decididas pela CJTEC, com base na aprovação de, pelo menos, dois terços de seus membros. 
Anexo C - Normas de Regulamentação da Comissão Julgadora do Título de Especialista em Cardiologia (CJTEC)

I. Definição

Comissão vinculada à diretoria científica da SBC, com a função de assessorá-la em assuntos referentes ao título de especialista e aos certificados de áreas de atuação.

II. Atribuições

1. Ser órgão de ligação entre AMB e SBC para assuntos relacionados ao título de especialista e áreas de atuação reconhecidas pela Comissão Mista de Especialidades -CME (AMB / CFM / CNRM).

2. Orientar e fiscalizar a emissão do TEC e dos certificados das áreas de atuação.

3. Estabelecer as diretrizes para obtenção e revalidação do TEC, de acordo com as normas da AMB

4. Analisar e colaborar na elaboração e aplicação da prova teórica, definindo seu conteúdo (anexo D).

5. Executar a prova prática para obtenção do TEC.

6. Analisar, regulamentar e pontuar as atividades científicas para fins de revalidação.

7. Coordenar o Curso Nacional de Reciclagem em Cardiologia, fiscalizando sua realização nos moldes propostos e estabelecendo seu programa (anexo E).

III. Constituição

1. Será constituída por 12 membros, com mandato de seis anos, sendo quatro representantes para cada macrorregião (Sudeste, Sul e Centro-Oeste; NorteNordeste) e ainda um componente da diretoria da SBC, de acordo com o estatuto.

2. Todos deverão ser portadores do TEC.

3. Haverá renovação de 1/3 dos seus componentes a cada dois anos, coincidindo com o mandato da diretoria da SBC.

4. Em caso de necessidade de substituição, um novo membro será indicado pelos integrantes da comissão e submetido à aprovação do presidente da SBC, para complementação do mandato, respeitando-se a região do membro substituído.

5. É vedada a recondução de um componente da comissão para um novo mandato consecutivo, ainda que o primeiro tenha sido em substituição.

IV. Organização

1. O coordenador da CJTEC terá um mandato de dois anos (os dois últimos), após ter cumprido 4 anos consecutivos como membro da comissão. Será indicado pela comissão e submetido à aprovação pelo presidente da SBC.

2. A comissão reunir-se-á ordinariamente 4 vezes ao ano:

A. em abril/maio, para avaliar a programação do calendário oficial da SBC e outros eventos para a revalidação do TEC;

B. em julho/agosto, para discutir e avaliar as questões da prova para obtenção do TEC;

C. em setembro/outubro, durante o Congresso da SBC, para fiscalizar a prova teórica e discutir assuntos pertinentes à comissão;

D. em outubro/novembro, para avaliar o calendário científico oficial da SBC e programar as provas práticas.

3. A comissão reunir-se-á extraordinariamente, desde que aprovado pela diretoria administrativa da SBC, quando necessário.

V. Disposições Gerais

1. As normas, regidas pelo estatuto da SBC, sobre o funcionamento da CJTEC também serão publicadas em separado no Jornal da SBC e ficarão a disposição no site da SBC, podendo ser modificadas somente por solicitação da CJTEC e com aprovação, por maioria simples, da diretoria da SBC.

2. Todos os procedimentos necessários para o cumprimento destas normas ficarão a cargo da Central de Eventos da SBC.

3. As presentes normas entrarão em vigor a partir da data de sua divulgação no site da SBC, quando então serão encaminhadas mensagens para todos os sócios; a divulgação incluirá outros "links" na rede mundial de computadores e o jornal da SBC. Posteriormente, serão publicadas nos Arquivos Brasileiros de Cardiologia - ABC

4. As situações aqui omissas serão julgadas e decididas pela CJTEC, com base na aprovação de pelo menos dois terços de seus membros.

\begin{tabular}{|c|c|}
\hline \multicolumn{2}{|c|}{ Anexo D - Os temas abordados nas questões da prova teórica seguirão a seguinte proporcionalidade } \\
\hline Fisiologia cardiovascular & $5 \%$ \\
\hline Semiologia cardiovascular & $5 \%$ \\
\hline Farmacologia cardiovascular & $7,5 \%$ \\
\hline Arritmias & $7,5 \%$ \\
\hline Doença coronariana aguda e crônica & $10 \%$ \\
\hline Dislipidemia e aterosclerose & $2,5 \%$ \\
\hline Valvopatias & $7,5 \%$ \\
\hline Hipertensão arterial & $7,5 \%$ \\
\hline Cardiopatias congênitas & $5 \%$ \\
\hline Doenças da aorta & $2,5 \%$ \\
\hline Exames complementares diagnósticos invasivos ou não & $10 \%$ \\
\hline Procedimentos cirúrgicos ou percutâneos & $5 \%$ \\
\hline Doenças pulmonares & $2,5 \%$ \\
\hline Endocardite infecciosa e febre reumática & $2,5 \%$ \\
\hline Cardiopatia e gravidez & $2,5 \%$ \\
\hline Emergências cardiovasculares & $2,5 \%$ \\
\hline Miocardiopatias & $2,5 \%$ \\
\hline Insuficiência cardíaca & $7,5 \%$ \\
\hline Doenças sistêmicas e o coração & $2,5 \%$ \\
\hline Reserva técnica & $2,5 \%$ \\
\hline
\end{tabular}

A prova será constituída de questões de conhecimento (30\%); condutas (40\%); interpretação e raciocínio (30\%), sendo que a empresa contratada para a confecção da prova deverá dar preferência às questões do tipo caso clínico, usando, sempre que necessário, o auxílio de imagens. 
Anexo E - Cada tópico deverá ser desenvolvido em forma de aula expositiva com duração de 30 minutos cada. O curso deverá ter a duração de cinco dias e ser contínuo. As aulas deverão ser baseadas nos livros textos consagrados e nas diretrizes da SBC. Não é um curso de atualização, é de reciclagem e também não é para falar da experiência pessoal, pois não é congresso ou simpósio.

Temas

1. Controle neural do coração e da circulação

2. Contratilidade e função de bomba do coração

3. Ciclo cardíaco e relação entre curvas de pressão, eventos mecânicos e acústicos

4. Semiogênese e fisiopatologia da dispnéia, do edema cardíaco e da cianose

5. Insuficiência cardíaca: fisiopatologia. eixo neuro-humoral

6. Insuficiência cardíaca: conceito, etiopatogenia e significado da classificação funcional e métodos de avaliação clínico laboratorial

7. Insuficiência cardíaca: critérios diagnóstico e métodos de avaliação clínico-laboratorial

8. Doppler-ecocardiografia nas pericardiopatias, cardiomiopatias e ICC

9. Insuficiência cardíaca: tratamento medicamentoso

10. Diretriz SBC-ICC: tópicos de sua importância

11. Cardiomiopatia: conceito, evolução clínica e prognóstico.

12. Cardiomiopatia chagásica: Diagnóstico e. forma indeterminada

13. Cardiomiopatia hipertrófica e restritiv: diagnóstic e evolução clínica

14. Estado de choque e choque cardiogênico: fisiopatologia e tratamento

15. Fisiopatologia e aspectos propedêuticos da dor torácica e cardíaca

16. Fluxo coronariano:. controle em situação normal e patológica

17. Fatores de risco coronariano:. critérios diagnósticos e tratamento Diretriz da SBC)

18. Patogenia da aterosclerose.

19. Etiopatogenia e fisiopatologia da insuficiência coronariana

20. Isquemia silenciosa. é possível detectar o inesperado?

21. Angina estável e variante: critérios, diagnósticos, classificação e prognóstico

22. Teste ergométrico: conceitos básicos e teorema de Bayes

23. Teste ergométrico (diretriz brasileira)

24. Diferenças do comportamento cardiovascular no idoso

25. Angina instável e infarto não $Q$ : novos tratamentos

26. Infarto agudo do miocárdio. conceito. diagnóstico em situações especiais e novos métodos enzimáticos

27. Diferenças entre o miocárdio "hibernado"e "atordoado"

28. Arritmias no IAM: algoritmo para o tratamento

29. Bloqueios no IAM: incidênci,. significado clínico e prognóstico

30. Infarto agudo do miocárdio: atendimento na emergência

31. Infarto agudo do miocárdio: tratamento medicamentoso

32. Cintilografia cardíaca, estudo da perfusã,. método:. indicações e tipos de avaliação e critérios

33. Doppler e ecocardiografia na hipertensão arterial, doença coronariana e infarto agudo do miocárdio

34. Interpretação da cinecoronariografia: ênfase em suas limitações

35. Angioplastia transluminal coronariana: indicações (Diretriz brasileira sobre angioplastia)

36. Tratamento cirúrgico da cardiopatia isquêmica

37. Hipertensão arteria:. conceito, etiopatogenia, classificação e critérios diagnósticos

38. Hipertensão arterial: prognóstico e epidemiologia

39. Hipertensão arterial: fisiopatologia e genética

40. Hipertensão arterial: tratamento (Diretriz brasileira)

41. Hipertensão arterial secundári:. diagnóstico diferencial; MAPA

42. Doenças da aorta e dissecção da aorta: fisiopatologia. diagnóstico clínico-laboratorial. Prognóstico

43. Tomografia computadorizada e ressonância nuclear magnética: quando utilizar?

44. Angina estável: os estudos comparativos entre os vários tratamentos
45. Diferenças de resposta à terapêutica no idoso

46. Cardiopatia na mulher (reposição hormonal)

47. Síndrome plurimetabólica/resistência à insulina

48. Epidemiologia clínica - os dados brasileiros da doença cardiovascular

49. Ressuscitação cardiopulmonar - básico e avançado

50. Cardiopatia congênita acianótica com hiperfluxo pulmonar.:tipos, manifestação clínica, diagnóstico, prognóstico, quando indicar tratamento cirúrgico?

51. Cardiopatia congênita cianótica com hiperfluxo pulmonar com ou sem hipertensão pulmonar:tipos de manifestação clínica, Diagnóstico, prognóstico, quando indicar tratamento cirúrgico?

52. Cardiopatia congênita acianótica obstrutiva direita e esquerda: tipos, manifestações clínicas, diagnóstico, prognóstico. quando indicar tratamento cirúrgico?

53. Cardiopatia congênita cianótica obstrutiva direita e esquerda:. tipos, manifestação clínica, diagnóstico, prognóstico. quando indicar tratamento cirúrgico?

54. Cardiologia intervencionista: tratamento das cardiopatias congênitas

55. Febre reumática: Conceito, etiopatogenia e critérios diagnósticos

56. Endocardite infecciosa: conceit,. etiopatogenia, fisiopatologia.

57. Estenose mitral: etiopatogenia, fisiopatologia, diagnóstico clínico, prognóstico

58. Insuficiência mitral: etiopatogenia, fisiopatologia, diagnóstico clínicolaboratórial, prognóstico

59. Prolapso de valva mitral: etiopatogenia, critérios diagnósticos, prognóstico, tratamento

60. Estenose aórtica: etiopatogenia, fisiopatologia, diagnóstico clínico-laboratorial, prognóstico

61. Insuficiência aórtica: Etiopatogenia, fisiopatologia, diagnóstico clínicolaboratórial, prognóstico

62. Doppler e ecocardiografia nas valvopatias e endocardite infecciosa: correlações hemodinâmicas e anatômicas

63. Tratamento das valvopatias mitral e aórtica por cateter

64. Tratamento cirúrgico da valvopatia mitral: cirurgia conservadora e troca valvar

65. Tratamento cirúrgico da valvopatia aórtica

66. Avaliação pré-operatória na cirurgia não cardíaca: fatores prognósticos e estratégias

67. Pericardite aguda e crônica: etiopatogenia, Fisiopatologia, Diagnóstico, complicações

68. Tromboembolismo pulmonar: etiopatogenia, diagnóstico, prognóstico,bases do tratamento

69. Cintilografia pulmonar: perfusão e inalação, métodos, indicações, critérios diagnósticos

70. Cor pulmonale crônico: etiopatogenia, fisiopatologia, diagnóstico, prognóstico

71. Bases eletrofisiológicas e etiológicas das arritmias cardíacas

72. Cardiopatia e gravidez - contra-indicações para a gravidez e cuidados com o tratamento durante a gestação

73. Hipotensão arterial, sincop:. conceito, fisiopatologia e diagnóstico diferencial

74. Bradicardia sinusal, doença no nó sinusal, e bloqueio AV: etiopatogenia, critérios diagnósticos e indicação de marcapasso, prognóstico

75. TPSV, pré-excitação, fibrilação flutter atrial: etiopatogenia. fisiopatologia,critérios diagnósticos, arritmias supraventriculares: tratamento farmacológico, ablação e cirurgia (Diretriz sbc)

76. Arritmias ventriculare: etiologia, Fisiopatologia, prognóstico, indicação do estudo eletrofisiológico, tratamento (Diretriz SBC)

77. Bloqueio de ramo: etiopatogenia,. critérios diagnósticos, prognóstico

78. Monitorização eletrocardiográfica ambulatorial: métodos, indicações, implicação diagnóstica 


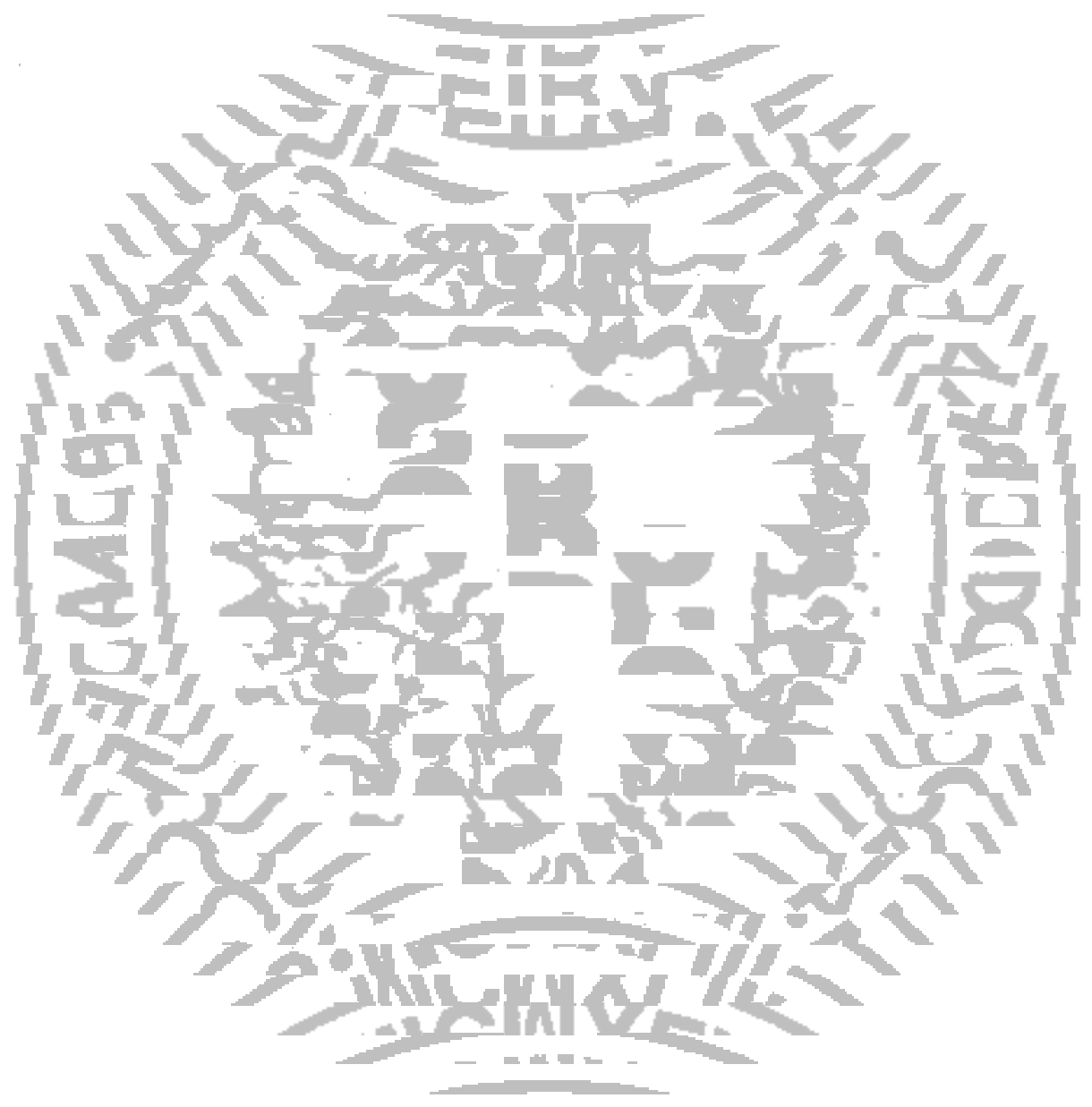

